\title{
The Lauricella functions and exact string scattering amplitudes
}

\author{
Sheng-Hong Lai, Jen-Chi Lee and Yi Yang \\ Department of Electrophysics, National Chiao Tung University, \\ 1001 University Street, Hsinchu, ROC \\ E-mail: xgcj944137@gmail.com, jcclee@cc.nctu.edu.tw, \\ yiyang@mail.nctu.edu.tw
}

ABSTRACT: We discover that the $26 D$ open bosonic string scattering amplitudes (SSA) of three tachyons and one arbitrary string state can be expressed in terms of the D-type Lauricella functions with associated $\operatorname{SL}(K+3, \mathbb{C})$ symmetry. As a result, SSA and symmetries or relations among SSA of different string states at various limits calculated previously can be rederived. These include the linear relations first conjectured by Gross [1-5] and later corrected and proved in [6-12] in the hard scattering limit, the recurrence relations in the Regge scattering limit with associated SL(5, C) symmetry [24-26] and the extended recurrence relations in the nonrelativistic scattering limit with associated $\mathrm{SL}(4, \mathbb{C})$ symmetry [29] discovered recently. Finally, as an application, we calculate a new recurrence relation of SSA which is valid for all energies.

Keywords: Bosonic Strings, Higher Spin Symmetry

ARXIV EPRINT: 1609.06014 


\section{Contents}

1 Introduction 1

2 Four-point string amplitudes $\quad 2$

3 Regge scattering limit $\quad 8$

4 Hard scattering limit $\quad 8$

5 Nonrelativistic scattering limit $\quad 11$

6 The associate symmetry group of string scattering amplitudes 13

\section{Introduction}

It has long been believed that there exist huge hidden spacetime symmetries of string theory. As a consistent theory of quantum gravity, string theory contains no free parameter and an infinite number of higher spin string states. On the other hand, the very soft exponential fall-off behavior of string scattering amplitudes (SSA) in the hard scattering limit, in contrast to the power law behavior of hard field theory scattering amplitudes, strongly suggests the existence of infinite number of relations among SSA of different string states. These relations or symmetries soften the UV structure of quantum string theory. Indeed, this kind of infinite relations were first conjectured by Gross [1-5] and later corrected and explicitly proved in [6-12] by using decoupling of zero-norm states (ZNS) [13$15]$, and can be used to reduce the number of independent hard SSA from $\infty$ down to 1 .

It was important to note that the linear relations obtained by decoupling of ZNS in the hard scattering limit corrected [6-9] the saddle point calculations of Gross [3, 4], Gross and Mende [1, 2] and Gross and Manes [5]. The results of the former authors were consistent with the decoupling of high energy ZNS or unitarity of the theory while those of the latter were not. See one simple example to be presented in eq. (4.3) in section 4. Independently, the inconsistency of the saddle point calculations of the above authors was also pointed out by the authors of [16] using the group theoretic approach of string amplitudes [17].

On the other hand, inspired by Witten's seminal paper [18], there have been tremendous developments on calculations of higher point and higher loop Yang-Mills and gravity field theory amplitudes [19]. Many interesting relations among these field theory amplitudes have also been proposed and suggested. In addition, connections between field theory and string theory amplitudes are currently under many investigations.

Historically, there were at least three approaches to probe stringy symmetries or relations among scattering amplitudes of higher spin string states. These include the gauge symmetry of Witten string field theory, the conjecture of Gross [3, 4] on symmetries or linear relations among SSA of different string states in the hard scattering limit by the saddle 
point method [1-5] and Moore's bracket algebra approach [20-22] of stringy symmetries. See a recent review [23] for some connections of these three approaches.

Recently, it was found that the Regge SSA of three tachyons and one arbitrary string states can be expressed in terms of a sum of Kummer functions $U$ [24-26], which soon later were shown to be the first Appell function $F_{1}$ [26]. Regge stringy symmetries or recurrence relations $[25,26]$ were then constructed and used to reduce the number of independent Regge SSA from $\infty$ down to 1. Moreover, an interesting link between Regge SSA and hard SSA was found [24, 27], and for each mass level the ratios among hard SSA can be extracted from Regge SSA. This result enables us to argue that the known $\mathrm{SL}(5 ; C)$ dynamical symmetry of the Appell function $F_{1}[28]$ is crucial to probe high energy spacetime symmetry of string theory.

More recently, the extended recurrence relations [29] among nonrelativistic low energy SSA of a class of string states with different spins and different channels were constructed by using the recurrence relations of the Gauss hypergeometric functions with associated $S L(4, \mathbb{C})$ symmetry [30]. These extended recurrence relations generalize and extend the field theory BCJ [31] relations to higher mass and higher spin string states.

To further uncover the structure of stringy symmetries, in section 2 of this paper we calculate the $26 D$ open bosonic SSA of three tachyons and one arbitrary string states at arbitrary energies. We discover that these SSA can be expressed in terms of the D-type Lauricella functions ${ }^{1}$ with associated $\operatorname{SL}(K+3, \mathbb{C})$ symmetry [30]. As a result, all these SSA and symmetries or relations among SSA of different string states at various limits calculated previously can be rederived. These will be presented in sections 3, 4 and 5 which include the recurrence relations in the Regge scattering limit $[25,26]$ with associated $\operatorname{SL}(5 ; C)$ symmetry, the linear relations conjectured by Gross $[3,4]$ and corrected and proved in [6$12]$ in the hard scattering limit and the extended recurrence relations in the nonrelativistic scattering limit $[29]$ with associated $\mathrm{SL}(4 ; C)$ symmetry discovered very recently. However, since not all Lauricella functions $F_{D}^{(K)}$ with arbitrary independent arguments can be used to represent SSA, it remained to be studied how the basis states of each $\mathrm{SL}(K+3, \mathbb{C})$ group representation for a given $K$ relates to SSA [33].

As a byproduct from the calculation of rederiving linear relations in the hard scattering limit directly from Lauricella functions, we propose an identity eq. (4.12) which generalizes the Stirling number identity eq. (4.13) [24, 27] used previously to extract ratios among hard SSA from the Appell functions in Regge SSA. Finally, as an example, in section 6 we calculate a new recurrence relation of SSA which is valid for all energies.

\section{Four-point string amplitudes}

We will consider SSA of three tachyons and one arbitrary string states put at the second vertex. For the 26D open bosonic string, the general states at mass level $M_{2}^{2}=2(N-1)$, $N=\sum_{n, m, l>0}\left(n r_{n}^{T}+m r_{m}^{P}+l r_{l}^{L}\right)$ with polarizations on the scattering plane are of the form

$$
\left|r_{n}^{T}, r_{m}^{P}, r_{l}^{L}\right\rangle=\prod_{n>0}\left(\alpha_{-n}^{T}\right)^{r_{n}^{T}} \prod_{m>0}\left(\alpha_{-m}^{P}\right)^{r_{m}^{P}} \prod_{l>0}\left(\alpha_{-l}^{L}\right)^{r_{l}^{L}}|0, k\rangle .
$$

\footnotetext{
${ }^{1}$ It is interesting to note that Lauricella function has been mentioned in [32] for the six gluon amplitude in superstring theory.
} 
In the CM frame, the kinematics are defined as

$$
\begin{aligned}
& k_{1}=\left(\sqrt{M_{1}^{2}+\left|\overrightarrow{k_{1}}\right|^{2}},-\left|\overrightarrow{k_{1}}\right|, 0\right), \\
& k_{2}=\left(\sqrt{M_{2}^{2}+\left|\overrightarrow{k_{1}}\right|^{2}},+\left|\overrightarrow{k_{1}}\right|, 0\right), \\
& k_{3}=\left(-\sqrt{M_{3}^{2}+\left|\overrightarrow{k_{3}}\right|^{2}},-\left|\overrightarrow{k_{3}}\right| \cos \phi,-\left|\overrightarrow{k_{3}}\right| \sin \phi\right), \\
& k_{4}=\left(-\sqrt{M_{4}^{2}+\left|\overrightarrow{k_{3}}\right|^{2}},+\left|\overrightarrow{k_{3}}\right| \cos \phi,+\left|\overrightarrow{k_{3}}\right| \sin \phi\right)
\end{aligned}
$$

with $M_{1}^{2}=M_{3}^{2}=M_{4}^{2}=-2$ and $\phi$ is the scattering angle. The Mandelstam variables are $s=-\left(k_{1}+k_{2}\right)^{2}, t=-\left(k_{2}+k_{3}\right)^{2}$ and $u=-\left(k_{1}+k_{3}\right)^{2}$. There are three polarizations on the scattering plane

$$
\begin{aligned}
e^{T} & =(0,0,1), \\
e^{L} & =\frac{1}{M_{2}}\left(\left|\overrightarrow{k_{1}}\right|, \sqrt{M_{2}^{2}+\left|\overrightarrow{k_{1}}\right|^{2}}, 0\right), \\
e^{P} & =\frac{1}{M_{2}}\left(\sqrt{M_{2}^{2}+\left|\overrightarrow{k_{1}}\right|^{2}},\left|\overrightarrow{k_{1}}\right|, 0\right) .
\end{aligned}
$$

For later use, we define

$$
k_{i}^{X}=e^{X} \cdot k_{i} \text { for } X=(T, P, L) .
$$

Note that SSA of three tachyons and one arbitrary string state with polarizations orthogonal to the scattering plane vanish.

For illustration, we begin with a simple case, namely, four-point function with the three tachyons and the highest spin state at mass level $M_{2}^{2}=2(N-1), N=p+q+r$ of the following form

$$
|p, q, r\rangle=\left(\alpha_{-1}^{T}\right)^{p}\left(\alpha_{-1}^{P}\right)^{q}\left(\alpha_{-1}^{L}\right)^{r}|0, k\rangle .
$$

The four-point scattering amplitude can be calculated as

$$
\begin{aligned}
A_{s t}^{(p, q, r)}= & \frac{\sin \left(\pi k_{2} \cdot k_{4}\right)}{\sin \left(\pi k_{1} \cdot k_{2}\right)} A_{t u}^{(p, q, r)}=\frac{\sin \left(\frac{u}{2}+2-N\right) \pi}{\sin \left(\frac{s}{2}+2-N\right) \pi} A_{t u}^{(p, q, r)} \\
= & \frac{(-1)^{N} \Gamma\left(\frac{s}{2}+2-N\right) \Gamma\left(-\frac{s}{2}-1+N\right)}{\Gamma\left(\frac{u}{2}+2\right) \Gamma\left(-\frac{u}{2}-1\right)} A_{t u}^{(p, q, r)} \\
= & \frac{(-1)^{N} \Gamma\left(\frac{s}{2}+2-N\right) \Gamma\left(-\frac{s}{2}-1+N\right)}{\Gamma\left(\frac{u}{2}+2\right) \Gamma\left(-\frac{u}{2}-1\right)} \\
& \times \int_{1}^{\infty} d x x^{k_{1} \cdot k_{2}}(x-1)^{k_{2} \cdot k_{3}} \cdot\left[\frac{k_{1}^{T}}{x}+\frac{k_{3}^{T}}{x-1}\right]^{p} \cdot\left[\frac{k_{1}^{P}}{x}+\frac{k_{3}^{P}}{x-1}\right]^{q} \cdot\left[\frac{k_{1}^{L}}{x}+\frac{k_{3}^{L}}{x-1}\right]^{r} \\
= & \frac{\Gamma\left(\frac{s}{2}+2-N\right) \Gamma\left(-\frac{s}{2}-1+N\right)}{\Gamma\left(\frac{u}{2}+2\right) \Gamma\left(-\frac{u}{2}-1\right)}\left(-k_{3}^{T}\right)^{p}\left(-k_{3}^{P}\right)^{q}\left(-k_{3}^{L}\right)^{r} \\
& \times \int_{1}^{\infty} d x x^{k_{1} \cdot k_{2}}(x-1)^{k_{2} \cdot k_{3}} \cdot\left[1-\left(-\frac{k_{1}^{T}}{k_{3}^{T}}\right) \frac{x-1}{x}\right]^{p} \cdot\left[1-\left(-\frac{k_{1}^{P}}{k_{3}^{P}}\right) \frac{x-1}{x}\right]^{q} \cdot\left[1-\left(-\frac{k_{1}^{L}}{k_{3}^{L}}\right) \frac{x-1}{x}\right]^{r} .
\end{aligned}
$$


In the above calculation, we have used the string BCJ relation

$$
A_{s t}^{(p, q, r)}=\frac{\sin \left(\pi k_{2} \cdot k_{4}\right)}{\sin \left(\pi k_{1} \cdot k_{2}\right)} A_{t u}^{(p, q, r)}
$$

which was proved by monodromy of integration of string amplitudes [34, 35] and explicitly proved recently in [29]. We can now do a change of variable $\frac{x-1}{x}=x^{\prime}$ to get

$$
\begin{aligned}
A_{s t}^{(p, q, r)}= & \frac{\Gamma\left(\frac{s}{2}+2-N\right) \Gamma\left(-\frac{s}{2}-1+N\right)}{\Gamma\left(\frac{u}{2}+2\right) \Gamma\left(-\frac{u}{2}-1\right)}\left(-k_{3}^{T}\right)^{p}\left(-k_{3}^{P}\right)^{q}\left(-k_{3}^{L}\right)^{r} \\
& \times \int_{0}^{1} d x^{\prime} x^{\prime \frac{-t}{2}-2}\left(1-x^{\prime}\right)^{\frac{-u}{2}-2}\left[1-\left(-\frac{k_{1}^{T}}{k_{3}^{T}}\right) x^{\prime}\right]^{p} \cdot\left[1-\left(-\frac{k_{1}^{P}}{k_{3}^{P}}\right) x^{\prime}\right]^{q} \cdot\left[1-\left(-\frac{k_{1}^{L}}{k_{3}^{L}}\right) x^{\prime}\right]^{r} \\
= & \frac{\Gamma\left(\frac{s}{2}+2-N\right) \Gamma\left(-\frac{s}{2}-1+N\right)}{\Gamma\left(\frac{u}{2}+2\right) \Gamma\left(-\frac{u}{2}-1\right)} \cdot\left(-k_{3}^{T}\right)^{p}\left(-k_{3}^{P}\right)^{q}\left(-k_{3}^{L}\right)^{r} \frac{\Gamma\left(-\frac{t}{2}-1\right) \Gamma\left(-\frac{u}{2}-1\right)}{\Gamma\left(\frac{s}{2}+2-N\right)} \\
& \times F_{D}^{(3)}\left(-\frac{t}{2}-1 ;-p,-q,-r ; \frac{s}{2}+2-N ; \frac{-k_{1}^{T}}{k_{3}^{T}}, \frac{-k_{1}^{P}}{k_{3}^{P}}, \frac{-k_{1}^{L}}{k_{3}^{L}}\right),
\end{aligned}
$$

which can be written as

$$
\begin{aligned}
A_{s t}^{(p, q, r)}= & \left(-k_{3}^{T}\right)^{p}\left(-k_{3}^{P}\right)^{q}\left(-k_{3}^{L}\right)^{r} \frac{\Gamma\left(-\frac{s}{2}-1+N\right) \Gamma\left(-\frac{t}{2}-1\right)}{\Gamma\left(\frac{u}{2}+2\right)} \\
& \times F_{D}^{(3)}\left(-\frac{t}{2}-1 ;-p,-q,-r ; \frac{s}{2}+2-N ;-C^{T},-C^{P},-C^{L}\right)
\end{aligned}
$$

if we define

$$
k_{i}^{X}=e^{X} \cdot k_{i}, \quad \frac{k_{1}^{X}}{k_{3}^{X}}=C^{X} .
$$

In eq. (2.13), the D-type Lauricella function $F_{D}^{(K)}$ is one of the four extensions of the Gauss hypergeometric function to $K$ variables and is defined as

$$
F_{D}^{(K)}\left(a ; b_{1}, \ldots, b_{K} ; c ; x_{1}, \ldots, x_{K}\right)=\sum_{n_{1}, \cdots, n_{K}} \frac{(a)_{n_{1}+\cdots+n_{K}}}{(c)_{n_{1}+\cdots+n_{K}}} \frac{\left(b_{1}\right)_{n_{1}} \cdots\left(b_{K}\right)_{n_{K}}}{n_{1} ! \cdots n_{K} !} x_{1}^{n_{1}} \cdots x_{K}^{n_{K}}
$$

where $(a)_{n}=a \cdot(a+1) \cdots(a+n-1)$ is the Pochhammer symbol. There is a integral representation of the Lauricella function $F_{D}^{(K)}$ discovered by Appell and Kampe de Feriet (1926) [36]

$$
\begin{aligned}
F_{D}^{(K)} & \left(a ; b_{1}, \ldots, b_{K} ; c ; x_{1}, \ldots, x_{K}\right) \\
\quad= & \frac{\Gamma(c)}{\Gamma(a) \Gamma(c-a)} \int_{0}^{1} d t t^{a-1}(1-t)^{c-a-1} \cdot\left(1-x_{1} t\right)^{-b_{1}}\left(1-x_{2} t\right)^{-b_{2}} \ldots\left(1-x_{K} t\right)^{-b_{K}},
\end{aligned}
$$

which can be used to directly calculate the amplitude in eq. (2.13). The relevance of the Lauricella function in eq. (2.16) for string scattering amplitudes was first suggested in [26]. 
We now calculate the string four-point scattering amplitude with three tachyons and one general higher spin state in eq. (2.1) as following

$$
\begin{aligned}
A_{s t}^{\left(p_{n} ; q_{m} ; r_{l}\right)}= & \frac{\sin \left(\pi k_{2} \cdot k_{4}\right)}{\sin \left(\pi k_{1} \cdot k_{2}\right)} A_{t u}^{\left(p_{n} ; q_{m} ; r_{l}\right)}=\frac{\sin \left(\frac{u}{2}+2-N\right) \pi}{\sin \left(\frac{s}{2}+2-N\right) \pi} A_{t u}^{\left(p_{n} ; q_{m} ; r_{l}\right)} \\
= & \frac{(-1)^{N} \Gamma\left(\frac{s}{2}+2-N\right) \Gamma\left(-\frac{s}{2}-1+N\right)}{\Gamma\left(\frac{u}{2}+2\right) \Gamma\left(-\frac{u}{2}-1\right)} \\
& \cdot \int_{1}^{\infty} d x x^{k_{1} \cdot k_{2}}(1-x)^{k_{2} \cdot k_{3}} \cdot \prod_{n=1}\left[\frac{(-1)^{n-1}(n-1) ! k_{1}^{T}}{x^{n}}+\frac{(-1)^{n-1}(n-1) ! k_{3}^{T}}{(x-1)^{n}}\right]^{p_{n}} \\
& \cdot \prod_{m=1}\left[\frac{(-1)^{m-1}(m-1) ! k_{1}^{P}}{x^{m}}+\frac{(-1)^{m-1}(m-1) ! k_{3}^{P}}{(x-1)^{m}}\right]^{q_{m}} \\
& \cdot \prod_{l=1}\left[\frac{(-1)^{l-1}(l-1) ! k_{1}^{L}}{x^{l}}+\frac{(-1)^{l-1}(l-1) ! k_{3}^{L}}{(x-1)^{l}}\right]^{r_{l}} \\
= & \frac{(-1)^{N} \Gamma\left(\frac{s}{2}+2-N\right) \Gamma\left(-\frac{s}{2}-1+N\right)}{\Gamma\left(\frac{u}{2}+2\right) \Gamma\left(-\frac{u}{2}-1\right)} \\
& \int_{1}^{\infty} d x x^{k_{1} \cdot k_{2}}(1-x)^{k_{2} \cdot k_{3}-N} \cdot \prod_{n=1}\left(k_{3}^{T}(-1)^{n-1}(n-1) !\left[1-\left(-\frac{k_{1}^{T}}{k_{3}^{T}}\right)\left(\frac{x-1}{x}\right)^{n}\right]\right)^{p_{n}} \\
& \cdot \prod_{m=1}\left(k_{3}^{P}(-1)^{m-1}(m-1) !\left[1-\left(-\frac{k_{1}^{P}}{k_{3}^{P}}\right)\left(\frac{x-1}{x}\right)^{m}\right]\right)^{q_{m}} \\
& \cdot \prod_{l=1}\left(k_{3}^{L}(-1)^{l-1}(l-1) !\left[1-\left(-\frac{k_{1}^{L}}{k_{3}^{L}}\right)\left(\frac{x-1}{x}\right)^{l}\right]\right)^{r_{l}} \cdot
\end{aligned}
$$

We can now do a change of variable $\frac{x-1}{x}=y$ to get

$$
\begin{aligned}
A_{s t}^{\left(p_{n} ; q_{m} ; r_{l}\right)=} & \frac{(-1)^{N} \Gamma\left(\frac{s}{2}+2-N\right) \Gamma\left(-\frac{s}{2}-1+N\right)}{\Gamma\left(\frac{u}{2}+2\right) \Gamma\left(-\frac{u}{2}-1\right)} \int_{0}^{1} d y y^{k_{2} \cdot k_{3}-N}(1-y)^{-k_{1} \cdot k_{2}-k_{2} \cdot k_{3}+N-2} \\
& \cdot \prod_{n=1}\left(k_{3}^{T}(-1)^{n-1}(n-1) !\left[1-\left(-\frac{k_{1}^{T}}{k_{3}^{T}}\right) y^{n}\right]\right)^{p_{n}} \\
& \cdot \prod_{m=1}\left(k_{3}^{P}(-1)^{m-1}(m-1) !\left[1-\left(-\frac{k_{1}^{P}}{k_{3}^{P}}\right) y^{m}\right]\right)^{q_{m}} \\
& \cdot \prod_{l=1}\left(k_{3}^{L}(-1)^{l-1}(l-1) !\left[1-\left(-\frac{k_{1}^{L}}{k_{3}^{L}}\right) y^{l}\right]\right)^{r_{l}} \\
= & \frac{(-1)^{N} \Gamma\left(\frac{s}{2}+2-N\right) \Gamma\left(-\frac{s}{2}-1+N\right)}{\Gamma\left(\frac{u}{2}+2\right) \Gamma\left(-\frac{u}{2}-1\right)} \cdot \prod_{n=1}\left[(-1)^{n-1}(n-1) ! k_{3}^{T}\right]^{p_{n}} \\
& \prod_{m=1}\left[(-1)^{m-1}(m-1) ! k_{3}^{P}\right]^{q_{m}} \prod_{l=1}\left[(-1)^{l-1}(l-1) ! k_{3}^{L}\right]^{r_{l}} \\
& \cdot \int_{0}^{1} d y y^{k_{2} \cdot k_{3}-N}(1-y)^{-k_{1} \cdot k_{2}-k_{2} \cdot k_{3}+N-2} \\
& \cdot\left[1-\left(z_{n}^{T} y\right)^{n}\right]^{p_{n}}\left[1-\left(z_{m}^{P} y\right)^{m}\right]^{q_{m}}\left[1-\left(z_{l}^{L} y\right)^{l}\right]^{r_{l}} .
\end{aligned}
$$


Finally the amplitude can be written in the following form

$$
\begin{aligned}
A_{s t}^{\left(p_{n} ; q_{m} ; r_{l}\right)}= & \frac{\Gamma\left(\frac{s}{2}+2-N\right) \Gamma\left(-\frac{s}{2}-1+N\right)}{\Gamma\left(\frac{u}{2}+2\right) \Gamma\left(-\frac{u}{2}-1\right)} \prod_{n=1}\left[-(n-1) ! k_{3}^{T}\right]^{p_{n}} \\
& \cdot \prod_{m=1}\left[-(m-1) ! k_{3}^{P}\right]^{q_{m}} \prod_{l=1}\left[-(l-1) ! k_{3}^{L}\right]^{r_{l}} \\
& \cdot \int_{0}^{1} d y y^{\frac{-t}{2}-2}(1-y)^{\frac{-u}{2}-2}\left[\left(1-z_{n}^{T} y\right)\left(1-z_{n}^{T} \omega_{n}^{2} y\right) \ldots\left(1-z_{n}^{T} \omega_{n}^{n-1} y\right)\right]^{p_{n}} \\
& \cdot\left[\left(1-z_{m}^{P} y\right)\left(1-z_{m}^{P} \omega_{m} y\right) \ldots\left(1-z_{m}^{P} \omega_{m}^{m-1} y\right)\right]^{q_{m}} \\
& \cdot\left[\left(1-z_{l}^{L} y\right)\left(1-z_{l}^{L} \omega_{l} y\right) \ldots\left(1-w_{l}^{L} \omega_{l}^{l-1} y\right)\right]^{p_{n}}
\end{aligned}
$$

which can then be written in terms of the D-type Lauricella function $F_{D}^{(K)}$ as following

$$
\begin{aligned}
A_{s t}^{\left(p_{n} ; q_{m} ; r_{l}\right)} & \\
= & \frac{\Gamma\left(\frac{s}{2}+2-N\right) \Gamma\left(-\frac{s}{2}-1+N\right)}{\Gamma\left(\frac{u}{2}+2\right) \Gamma\left(-\frac{u}{2}-1\right)} \frac{\Gamma\left(-\frac{t}{2}-1\right) \Gamma\left(-\frac{u}{2}-1\right)}{\Gamma\left(\frac{s}{2}+2-N\right)} \\
& \cdot \prod_{n=1}\left[-(n-1) ! k_{3}^{T}\right]^{p_{n}} \prod_{m=1}\left[-(m-1) ! k_{3}^{P}\right]^{q_{m}} \prod_{l=1}\left[-(l-1) ! k_{3}^{L}\right]^{r_{l}} \\
& \cdot F_{D}^{(K)}\left(\begin{array}{r}
-\frac{t}{2}-1 ;\left\{-p_{1}\right\}^{1}, \ldots,\left\{-p_{n}\right\}^{n},\left\{-q_{1}\right\}^{1}, \ldots,\left\{-q_{m}\right\}^{m},\left\{-r_{1}\right\}^{1}, \ldots,\left\{-r_{l}\right\}^{l} ; \frac{s}{2}+2-N ; \\
{\left[z_{1}^{T}\right], \ldots,\left[z_{n}^{T}\right],\left[z_{1}^{P}\right], \ldots,\left[z_{m}^{P}\right],\left[z_{1}^{L}\right], \ldots,\left[z_{l}^{L}\right],}
\end{array}\right) \\
= & \frac{\Gamma\left(-\frac{s}{2}-1+N\right) \Gamma\left(-\frac{t}{2}-1\right)}{\Gamma\left(\frac{u}{2}+2\right)} \prod_{n=1}\left[-(n-1) ! k_{3}^{T}\right]^{p_{n}} \prod_{m=1}\left[-(m-1) ! k_{3}^{P}\right]^{q_{m}} \prod_{l=1}\left[-(l-1) ! k_{3}^{L}\right]^{r_{l}} \\
& \cdot F_{D}^{(K)}\left(\begin{array}{c}
-\frac{t}{2}-1 ;\left\{-p_{1}\right\}^{1}, \ldots,\left\{-p_{n}\right\}^{n},\left\{-q_{1}\right\}^{1}, \ldots,\left\{-q_{m}\right\}^{m},\left\{-r_{1}\right\}^{1}, \ldots,\left\{-r_{l}\right\}^{l} ; \frac{s}{2}+2-N ; \\
{\left[z_{1}^{T}\right], \ldots,\left[z_{n}^{T}\right],\left[z_{1}^{P}\right], \ldots,\left[z_{m}^{P}\right],\left[z_{1}^{L}\right], \ldots,\left[z_{l}^{L}\right]}
\end{array}\right)
\end{aligned}
$$

where we have defined

$$
k_{i}^{X}=e^{X} \cdot k_{i}, \omega_{k}=e^{\frac{2 \pi i}{k}}, \quad z_{k}^{X}=\left(\frac{-k_{1}^{X}}{k_{3}^{X}}\right)^{\frac{1}{k}}
$$

and

$$
\{a\}^{n}=\underbrace{a, a, \cdots, a}_{n}, \quad\left[z_{k}^{X}\right]=z_{k}^{X}, z_{k}^{X} e^{\frac{2 \pi i}{k}}, \cdots, z_{k}^{X} e^{\frac{2 \pi i(k-1)}{k}} \text { or } z_{k}^{X}, z_{k}^{X} \omega_{k}, \ldots, z_{k}^{X} \omega_{k}^{k-1} .
$$

The integer $K$ in eq. (2.20) is defined to be

$$
K=\sum_{\substack{j=1 \\\left\{\text { for all } r_{j}^{T} \neq 0\right\}}}^{n} j+\sum_{\substack{j=1 \\\left\{\text { for all } r_{j}^{P} \neq 0\right\}}}^{m} j+\sum_{\substack{j=1 \\\left\{\text { for all } r_{j}^{L} \neq 0\right\}}}^{l} j .
$$

For a given $K$, there can be SSA with different mass level $N$. 
Alternatively, by using the identity of Lauricella function for $b_{i} \in Z^{-}$

$$
\begin{aligned}
F_{D}^{(K)}\left(a ; b_{1}, \ldots, b_{K} ; c ; x_{1}, \ldots, x_{K}\right)= & \frac{\Gamma(c) \Gamma\left(c-a-\sum b_{i}\right)}{\Gamma(c-a) \Gamma\left(c-\sum b_{i}\right)} \\
& \cdot F_{D}^{(K)}\left(a ; b_{1}, \ldots, b_{K} ; 1+a+\sum b_{i}-c ; 1-x_{1}, \ldots, 1-x_{K}\right),
\end{aligned}
$$

we can rederive the string BCJ relation $[29,34,35]$

$$
\frac{A_{s t}^{\left(r_{n}^{T}, r_{m}^{P}, r_{l}^{L}\right)}}{A_{t u}^{\left(r_{n}^{T}, r_{m}^{P}, r_{l}^{L}\right)}}=\frac{(-)^{N} \Gamma\left(-\frac{s}{2}-1\right) \Gamma\left(\frac{s}{2}+2\right)}{\Gamma\left(\frac{u}{2}+2-N\right) \Gamma\left(-\frac{u}{2}-1+N\right)}=\frac{\sin \left(\frac{\pi u}{2}\right)}{\sin \left(\frac{\pi s}{2}\right)}=\frac{\sin \left(\pi k_{2} \cdot k_{4}\right)}{\sin \left(\pi k_{1} \cdot k_{2}\right)}
$$

which gives another form of the $(s, t)$ channel amplitude

$$
\begin{aligned}
& A_{s t}^{\left(r_{n}^{T}, r_{m}^{P}, r_{l}^{L}\right)} \\
& =B\left(-\frac{t}{2}-1,-\frac{s}{2}-1\right) \prod_{n=1}\left[-(n-1) ! k_{3}^{T}\right]^{r_{n}^{T}} \cdot \prod_{m=1}\left[-(m-1) ! k_{3}^{P}\right]^{r_{m}^{P}} \prod_{l=1}\left[-(l-1) ! k_{3}^{L}\right]^{r_{l}^{L}} \\
& \quad \cdot F_{D}^{(K)}\left(-\frac{t}{2}-1 ; R_{n}^{T}, R_{m}^{P}, R_{l}^{L} ; \frac{u}{2}+2-N ; \tilde{Z}_{n}^{T}, \tilde{Z}_{m}^{P}, \tilde{Z}_{l}^{L}\right)
\end{aligned}
$$

and similarly the $(t, u)$ channel amplitude

$$
\begin{aligned}
A_{t u}^{\left(r_{n}^{T}, r_{m}^{P}, r_{l}^{L}\right)} & \\
= & B\left(-\frac{t}{2}-1,-\frac{u}{2}-1\right) \prod_{n=1}\left[-(n-1) ! k_{3}^{T}\right]^{r_{n}^{T}} \cdot \prod_{m=1}\left[-(m-1) ! k_{3}^{P}\right]^{r_{m}^{P}} \prod_{l=1}\left[-(l-1) ! k_{3}^{L}\right]^{r_{l}^{L}} \\
\quad & F_{D}^{(K)}\left(-\frac{t}{2}-1 ; R_{n}^{T}, R_{m}^{P}, R_{l}^{L} ; \frac{s}{2}+2-N ; Z_{n}^{T}, Z_{m}^{P}, Z_{l}^{L}\right) .
\end{aligned}
$$

In eq. (2.26) and eq. (2.27), we have defined

$$
R_{k}^{X}=\left\{-r_{1}^{X}\right\}^{1}, \cdots,\left\{-r_{k}^{X}\right\}^{k} \text { with }\{a\}^{n}=\underbrace{a, a, \cdots, a}_{n},
$$

and

$$
Z_{k}^{X}=\left[z_{1}^{X}\right], \cdots,\left[z_{k}^{X}\right] \text { with }\left[z_{k}^{X}\right]=z_{k 0}^{X}, \cdots, z_{k(k-1)}^{X}
$$

where

$$
z_{k}^{X}=\left|\left(-\frac{k_{1}^{X}}{k_{3}^{X}}\right)^{\frac{1}{k}}\right|, z_{k k^{\prime}}^{X}=z_{k}^{X} e^{\frac{2 \pi i k^{\prime}}{k}}, \tilde{z}_{k k^{\prime}}^{X}=1-z_{k k^{\prime}}^{X}
$$

for $k^{\prime}=0, \cdots, k-1$.

With the notation introduced above, the $(s, t)$ channel amplitude in eq. (2.20) can be rewritten as

$$
\begin{aligned}
A_{s t}^{\left(r_{n}^{T}, r_{m}^{P}, r_{l}^{L}\right)} & \\
= & B\left(-\frac{t}{2}-1,-\frac{s}{2}-1+N\right) \prod_{n=1}\left[-(n-1) ! k_{3}^{T}\right]^{r_{n}^{T}} \cdot \prod_{m=1}\left[-(m-1) ! k_{3}^{P}\right]^{r_{m}^{P}} \prod_{l=1}\left[-(l-1) ! k_{3}^{L}\right]^{r_{l}^{L}} \\
& \cdot F_{D}^{(K)}\left(-\frac{t}{2}-1 ; R_{n}^{T}, R_{m}^{P}, R_{l}^{L} ; \frac{s}{2}+2-N ; Z_{n}^{T}, Z_{m}^{P}, Z_{l}^{L}\right) .
\end{aligned}
$$




\section{$3 \quad$ Regge scattering limit}

With the exact SSA calculated in eq. (2.31), eq. (2.26) and eq. (2.27) which are valid for all kinematic regimes, we can rederive SSA and symmetries or relations among SSA of different string states at various limits calculated previously. These include the linear relations conjectured by Gross [1-5] and proved in [6-12] in the hard scattering limit, the recurrence relations in the Regge scattering limit [24-26] and the extended recurrence relations in the nonrelativistic scattering limit [29] discovered recently. In this section, we first calculate the Regge scattering limit. The relevant kinematics in Regge limit are

$$
\begin{aligned}
& k_{1}^{T}=0, \quad k_{3}^{T} \simeq-\sqrt{-t}, \\
& k_{1}^{P} \simeq-\frac{s}{2 M_{2}}, k_{3}^{P} \simeq-\frac{\tilde{t}}{2 M_{2}}=-\frac{t-M_{2}^{2}-M_{3}^{2}}{2 M_{2}}, \\
& k_{1}^{L} \simeq-\frac{s}{2 M_{2}}, k_{3}^{L} \simeq-\frac{\tilde{t}^{\prime}}{2 M_{2}}=-\frac{t+M_{2}^{2}-M_{3}^{2}}{2 M_{2}} .
\end{aligned}
$$

One can easily calculate

$$
\tilde{z}_{k k^{\prime}}^{T}=1, \tilde{z}_{k k^{\prime}}^{P}=1-\left(-\frac{s}{\tilde{t}}\right)^{1 / k} e^{\frac{i 2 \pi k^{\prime}}{k}} \sim s^{1 / k}
$$

and

$$
\tilde{z}_{k k^{\prime}}^{L}=1-\left(-\frac{s}{\tilde{t}^{\prime}}\right)^{1 / k} e^{\frac{i 2 \pi k^{\prime}}{k}} \sim s^{1 / k} .
$$

In the Regge limit, the SSA in eq. (2.26) reduces to

$$
\begin{aligned}
& A_{s t}^{\left(r_{n}^{T}, r_{m}^{P}, r_{l}^{L}\right)} \\
& \simeq B\left(-\frac{t}{2}-1,-\frac{s}{2}-1\right) \prod_{n=1}[(n-1) ! \sqrt{-t}]^{r_{n}^{T}} \cdot \prod_{m=1}\left[(m-1) ! \frac{\tilde{t}}{2 M_{2}}\right]^{r_{m}^{P}} \prod_{l=1}\left[(l-1) ! \frac{\tilde{t}^{\prime}}{2 M_{2}}\right]^{r_{l}^{L}} \\
& \quad \cdot F_{1}\left(-\frac{t}{2}-1 ;-q_{1},-r_{1} ;-\frac{s}{2} ; \frac{s}{\tilde{t}}, \frac{s}{\tilde{t}^{\prime}}\right) .
\end{aligned}
$$

where $F_{1}$ is the Appell function. Eq. (3.6) agrees with the result obtained in [26] previously.

\section{Hard scattering limit}

In this section, we rederive the linear relations conjectured by Gross [1-5] and corrected and proved in [6-12] in the hard scattering limit. As we will see that the calculation will be more subtle than that of the Regge scattering limit. In the hard scattering limit $e^{P}=e^{L}[6-8]$, and we can consider only the polarization $e^{L}$ case. We first briefly review the results [23] for linear relations among hard SSA. One first observes that for each fixed mass level $N$ only states of the following form $[10,11]$

$$
|N, 2 m, q\rangle=\left(\alpha_{-1}^{T}\right)^{N-2 m-2 q}\left(\alpha_{-1}^{L}\right)^{2 m}\left(\alpha_{-2}^{L}\right)^{q}|0, k\rangle
$$

are of leading order in energy in the HSS limit. The choice of only even power $2 m$ in $\alpha_{-1}^{L}$ is the result of the observation [6-8] that the naive energy order of the amplitudes will in 
general drop by even number of energy powers. Scattering amplitudes corresponding to states with $\left(\alpha_{-1}^{L}\right)^{2 m+1}$ turn out to be of subleading order in energy. Many simplifications occur if we apply Ward identities or decoupling of ZNS only on high energy states in eq. (4.1) in the HSS limit. One important result was the discovery of the linear relations among hard SSA of different string states at each fixed mass level $N[10,11]$

$$
\frac{A_{s t}^{(N, 2 m, q)}}{A_{s t}^{(N, 0,0)}}=\left(-\frac{1}{M_{2}}\right)^{2 m+q}\left(\frac{1}{2}\right)^{m+q}(2 m-1) ! ! .
$$

Exactly the same results can also be obtained by two other calculations, the Virasoro constraint calculation and the corrected saddle-point calculation $[10,11]$. In the decoupling of ZNS calculations at the mass level $M_{2}^{2}=4$, for example, there are four leading order SSA [6-8]

$$
A_{T T T}: A_{L L T}: A_{(L T)}: A_{[L T]}=8: 1:-1:-1
$$

which are proportional to each other. While the saddle point calculation of [5] gave $A_{T T T} \propto$ $A_{[L T]}$, and $A_{L L T}=0$ which are inconsistent with the decoupling of ZNS or unitarity of the theory. Indeed, a sample calculation was done [6-8] to explicitly verify the ratios in eq. (4.3).

One interesting application of eq. (4.2) was the derivation of relation of $A_{s t}^{(N, 2 m, q)}$ and $A_{t u}^{(N, 2 m, q)}$ in the hard scattering limit [37]

$$
A_{s t}^{(N, 2 m, q)} \simeq(-)^{N} \frac{\sin \left(\pi k_{2} \cdot k_{4}\right)}{\sin \left(\pi k_{1} \cdot k_{2}\right)} A_{t u}^{(N, 2 m, q)}
$$

where

$$
\begin{aligned}
A_{t u}^{(N, 2 m, q)} \simeq & \sqrt{\pi}(-1)^{N-1} 2^{-N} E^{-1-2 N}\left(\sin \frac{\phi}{2}\right)^{-3}\left(\cos \frac{\phi}{2}\right)^{5-2 N} \\
& \cdot \exp \left[-\frac{t \ln t+u \ln u-(t+u) \ln (t+u)}{2}\right]
\end{aligned}
$$

Eq. (4.4) was shown to be valid for scatterings of four arbitrary string states and was obtained in $2006,{ }^{2}$ and thus was earlier than the discovery of four point field theory BCJ relations [31] and "string BCJ relations" in eq. (2.25) [29, 34, 35]. In contrast to the calculation of string BCJ relations [34, 35] which was motivated by the field theory BCJ relations [31], the derivation of eq. (4.4) was motivated by the calculation of hard closed SSA [37] by using KLT relation [38]. See a more detailed discussion in a recent publication [29].

\footnotetext{
${ }^{2}$ There was an $(-)^{N}$ ambiguity in eq. (42) in the high energy calculation of $(s ; t)$ channel amplitude in ref. [37] due to the pole structure of the gamma function $\Gamma(x)$ for negative real $x$.
} 
We are now ready to rederive eq. (4.1) and eq. (4.2) from eq. (2.26). The relevant kinematics are

$$
\begin{aligned}
& k_{1}^{T}=0, \quad k_{3}^{T} \simeq-E \sin \phi, \\
& k_{1}^{L} \simeq-\frac{2 p^{2}}{M_{2}} \simeq-\frac{2 E^{2}}{M_{2}}, \\
& k_{3}^{L} \simeq \frac{2 E^{2}}{M_{2}} \sin ^{2} \frac{\phi}{2} .
\end{aligned}
$$

One can calculate

$$
\tilde{z}_{k k^{\prime}}^{T}=1, \tilde{z}_{k k^{\prime}}^{L}=1-\left(-\frac{s}{t}\right)^{1 / k} e^{\frac{i 2 \pi k^{\prime}}{k}} \sim O(1) .
$$

The SSA in eq. (2.26) reduces to

$$
\begin{aligned}
A_{s t}^{\left(r_{n}^{T}, r_{l}^{L}\right)}= & B\left(-\frac{t}{2}-1,-\frac{s}{2}-1\right) \cdot \prod_{n=1}[(n-1) ! E \sin \phi]^{r_{n}^{T}} \prod_{l=1}\left[-(l-1) ! \frac{2 E^{2}}{M_{2}} \sin ^{2} \frac{\phi}{2}\right]^{r_{l}^{L}} \\
& \cdot F_{D}^{(K)}\left(-\frac{t}{2}-1 ; R_{n}^{T}, R_{l}^{L} ; \frac{u}{2}+2-N ;(1)_{n}, \tilde{Z}_{l}^{L}\right) .
\end{aligned}
$$

As was mentioned above that, in the hard scattering limit, there was a difference between the naive energy order and the real energy order corresponding to the $\left(\alpha_{-1}^{L}\right)^{r_{1}^{L}}$ operator in eq. (2.1). So let's pay attention to the corresponding summation and write

$$
\begin{aligned}
A_{s t}^{\left(r_{n}^{T}, r_{l}^{L}\right)}= & B\left(-\frac{t}{2}-1,-\frac{s}{2}-1\right) \cdot \prod_{n=1}[(n-1) ! E \sin \phi]^{r_{n}^{T}} \prod_{l=1}\left[-(l-1) ! \frac{2 E^{2}}{M_{2}} \sin ^{2} \frac{\phi}{2}\right]^{r_{l}^{L}} \\
& \cdot \sum_{k_{r}} \frac{\left(-\frac{t}{2}-1\right)_{k_{r}}}{\left(\frac{u}{2}+2-N\right)_{k_{r}}} \frac{\left(-r_{1}^{L}\right)_{k_{r}}}{k_{r} !}\left(1+\frac{s}{t}\right)^{k_{r}} \cdot(\cdots)
\end{aligned}
$$

where we have used $(a)_{n+m}=(a)_{n}(a+n)_{m}$ and $(\cdots)$ are terms which are not relevant to the following discussion. We then propose the following formula

$$
\begin{aligned}
& \sum_{k_{r}=0}^{r_{1}^{L}} \frac{\left(-\frac{t}{2}-1\right)_{k_{r}}}{\left(\frac{u}{2}+2-N\right)_{k_{r}}} \frac{\left(-r_{1}^{L}\right)_{k_{r}}}{k_{r} !}\left(1+\frac{s}{t}\right)^{k_{r}} \\
& \quad=0 \cdot\left(\frac{t u}{s}\right)^{0}+0 \cdot\left(\frac{t u}{s}\right)^{-1}+\cdots+0 \cdot\left(\frac{t u}{s}\right)^{-\left[\frac{r_{1}^{L}+1}{2}\right]-1}+C_{r_{1}^{L}}\left(\frac{t u}{s}\right)^{-\left[\frac{r_{1}^{L}+1}{2}\right]}+O\left\{\left(\frac{t u}{s}\right)^{-\left[\frac{r_{1}^{L}+1}{2}\right]+1}\right\} .
\end{aligned}
$$

where $C_{r_{1}^{L}}$ is independent of energy $E$ and depends on $r_{1}^{L}$ and possibly scattering angle $\phi$. For $r_{1}^{L}=2 m$ being an even number, we further propose that $C_{r_{1}^{L}}=\frac{(2 m) !}{m !}$ and is $\phi$ independent. We have verified eq. (4.12) for $r_{1}^{L}=0,1,2, \cdots, 10$. 
It should be noted that, taking Regge limit $\left(s \rightarrow \infty\right.$ with $t$ fixed) and setting $r_{1}^{L}=2 m$, eq. (4.12) reduces to the Stirling number identity,

$$
\begin{aligned}
& \sum_{k_{r}=0}^{2 m} \frac{\left(-\frac{t}{2}-1\right)_{k_{r}}}{\left(-\frac{s}{2}\right)_{k_{r}}} \frac{(-2 m)_{k_{r}}}{k_{r} !}\left(\frac{s}{t}\right)^{k_{r}} \simeq \sum_{k_{r}=0}^{2 m}(-2 m)_{k_{r}}\left(-\frac{t}{2}-1\right)_{k_{r}} \frac{(-2 / t)^{k_{r}}}{k_{r} !} \\
& \quad=0 \cdot(-t)^{0}+0 \cdot(-t)^{-1}+\cdots+0 \cdot(-t)^{-m+1}+\frac{(2 m) !}{m !}(-t)^{-m}+O\left\{\left(\frac{1}{t}\right)^{m+1}\right\},
\end{aligned}
$$

which was proposed in [24] and proved in [27].

It was demonstrated in [24] that the ratios in the hard scattering limit in eq. (4.2) can be reproduced from a class of Regge string scattering amplitudes presented in eq. (3.6). The key of the mathematical proof [27] was the new Stirling number identity proposed in eq. (4.13).

In eq. (4.12), the 0 terms correspond to the naive leading energy orders in the hard SSA calculation. The true leading order SSA in the hard scattering limit can then be identified

$$
\begin{aligned}
A_{s t}^{\left(r_{n}^{T}, r_{l}^{L}\right)} \simeq & B\left(-\frac{t}{2}-1,-\frac{s}{2}-1\right) \cdot \prod_{n=1}[(n-1) ! E \sin \phi]^{r_{n}^{T}} \prod_{l=1}\left[-(l-1) ! \frac{2 E^{2}}{M_{2}} \sin ^{2} \frac{\phi}{2}\right]^{r_{l}^{L}} \\
& \cdot C_{r_{1}^{L}}(E \sin \phi)^{-2\left[\frac{r_{1}^{L}+1}{2}\right]} \cdot(\cdots) \\
\sim & E^{N-\sum_{n \geq 2} n r_{n}^{T}-\left(2\left[\frac{r_{1}^{L}+1}{2}\right]-r_{1}^{L}\right)-\sum_{l \geq 3} l r_{l}^{L}}
\end{aligned}
$$

which means that SSA reaches its highest energy when $r_{n \geq 2}^{T}=r_{l \geq 3}^{L}=0$ and $r_{1}^{L}=2 m$ being an even number. This is consistent with the previous result presented in eq. (4.1) [6-12].

Finally, the leading order SSA in the hard scattering limit, i.e. $r_{1}^{T}=N-2 m-2$, $r_{1}^{L}=2 m$ and $r_{2}^{L}=q$, can be calculated to be

$$
\begin{aligned}
A_{s t}^{(N-2 m-2 q, 2 m, q)} & \simeq B\left(-\frac{t}{2}-1,-\frac{s}{2}-1\right)(E \sin \phi)^{N} \frac{(2 m) !}{m !}\left(-\frac{1}{2 M_{2}}\right)^{2 m+q} \\
& =(2 m-1) ! !\left(-\frac{1}{M_{2}}\right)^{2 m+q}\left(\frac{1}{2}\right)^{m+q} A_{s t}^{(N, 0,0)}
\end{aligned}
$$

which reproduces the ratios in eq. (4.2), and is consistent with the previous result [6-12].

\section{$5 \quad$ Nonrelativistic scattering limit}

In a recent paper [29] both $s-t$ and $t-u$ channel nonrelativistic low energy string scattering amplitudes of three tachyons and one leading trajectory string state at arbitrary mass levels were calculated. It was discovered that the mass and spin dependent nonrelativistic string BCJ relations $[34,35]$ can be expressed in terms of Gauss hypergeometric functions. As an application, for each fixed mass level $N$, the extended recurrence relations among 
nonrelativistic low energy string scattering amplitudes of string states with different spins and different channels can be derived.

In this section, we intend to rederive the results stated above from the Lauricella functions. In the nonrelativistic limit $\left|\overrightarrow{k_{1}}\right| \ll M_{2}$, we have

$$
\begin{aligned}
& k_{1}^{T}=0, k_{3}^{T}=-\left[\frac{\epsilon}{2}+\frac{\left(M_{1}+M_{2}\right)^{2}}{4 M_{1} M_{2} \epsilon}\left|\overrightarrow{k_{1}}\right|^{2}\right] \sin \phi, \\
& k_{1}^{L}=-\frac{M_{1}+M_{2}}{M_{2}}\left|\overrightarrow{k_{1}}\right|+O\left(\left|\overrightarrow{k_{1}}\right|^{2}\right), \\
& k_{3}^{L}=-\frac{\epsilon}{2} \cos \phi+\frac{M_{1}+M_{2}}{2 M_{2}}\left|\overrightarrow{k_{1}}\right|+O\left(\left|\overrightarrow{k_{1}}\right|^{2}\right), \\
& k_{1}^{P}=-M_{1}+O\left(\left|\overrightarrow{k_{1}}\right|^{2}\right) \\
& k_{3}^{P}=\frac{M_{1}+M_{2}}{2}-\frac{\epsilon}{2 M_{2}} \cos \phi\left|\overrightarrow{k_{1}}\right|+O\left(\left|\overrightarrow{k_{1}}\right|^{2}\right)
\end{aligned}
$$

where $\epsilon=\sqrt{\left(M_{1}+M_{2}\right)^{2}-4 M_{3}^{2}}$. One can easily calculate

$$
z_{k}^{T}=z_{k}^{L}=0, z_{k}^{P} \simeq\left|\left(\frac{2 M_{1}}{M_{1}+M_{2}}\right)^{\frac{1}{k}}\right| .
$$

The SSA in eq. (2.31) reduces to

$$
\begin{aligned}
A_{s t}^{\left(r_{n}^{T}, r_{m}^{P}, r_{l}^{L}\right)} \simeq & \prod_{n=1}\left[(n-1) ! \frac{\epsilon}{2} \sin \phi\right]^{r_{n}^{T}} \prod_{m=1}\left[-(m-1) ! \frac{M_{1}+M_{2}}{2}\right]^{r_{m}^{P}} \\
& \cdot \prod_{l=1}\left[(l-1) ! \frac{\epsilon}{2} \cos \phi\right]^{r_{l}^{L}} B\left(\frac{M_{1} M_{2}}{2}, 1-M_{1} M_{2}\right) \\
& \cdot F_{D}^{(K)}\left(\frac{M_{1} M_{2}}{2} ; R_{m}^{P} ; M_{1} M_{2} ;\left(\frac{2 M_{1}}{M_{1}+M_{2}}\right)_{m}\right)
\end{aligned}
$$

where

$$
K=\sum_{\substack{\left.j=1 \\ \text { for all } r_{j}^{P} \neq 0\right\}}}^{m} j
$$

Note that for string states with $r_{k}^{P}=0$ for all $k \geq 2$, one has $K=1$ and the Lauricella functions in the low energy nonrelativistic SSA reduce to the Gauss hypergeometric functions $F_{D}^{(1)}={ }_{2} F_{1}$. In particular, for the case of $r_{1}^{T}=N_{1}, r_{1}^{P}=N_{3}, r_{1}^{L}=N_{2}$, and $r_{k}^{X}=0$ for all $k \geq 2$, the SSA reduces to

$$
\begin{aligned}
A_{s t}^{\left(N_{1}, N_{2}, N_{3}\right)}= & \left(\frac{\epsilon}{2} \sin \phi\right)^{N_{1}}\left(\frac{\epsilon}{2} \cos \phi\right)^{N_{2}} \cdot\left(-\frac{M_{1}+M_{2}}{2}\right)^{N_{3}} B\left(\frac{M_{1} M_{2}}{2}, 1-M_{1} M_{2}\right) \\
& \cdot{ }_{2} F_{1}\left(\frac{M_{1} M_{2}}{2} ;-N_{3} ; M_{1} M_{2} ; \frac{2 M_{1}}{M_{1}+M_{2}}\right)
\end{aligned}
$$


which agrees with the result obtained in [29] previously. Similarly, one can calculate the corresponding nonrelativistic $t-u$ channel amplitude as

$$
\begin{aligned}
A_{t u}^{\left(N_{1}, N_{2}, N_{3}\right)}= & (-1)^{N}\left(\frac{\epsilon}{2} \sin \phi\right)^{N_{1}}\left(\frac{\epsilon}{2} \cos \phi\right)^{N_{2}} \cdot\left(-\frac{M_{1}+M_{2}}{2}\right)^{N_{3}} B\left(\frac{M_{1} M_{2}}{2}, \frac{M_{1} M_{2}}{2}\right) \\
& \cdot{ }_{2} F_{1}\left(\frac{M_{1} M_{2}}{2} ;-N_{3} ; M_{1} M_{2} ; \frac{2 M_{1}}{M_{1}+M_{2}}\right) .
\end{aligned}
$$

Finally the ratio of $s-t$ and $t-u$ channel amplitudes is [29]

$$
\begin{aligned}
\frac{A_{s t}^{(p, r, q)}}{A_{t u}^{(p, r, q)}} & =(-1)^{N} \frac{B\left(-M_{1} M_{2}+1, \frac{M_{1} M_{2}}{2}\right)}{B\left(\frac{M_{1} M_{2}}{2}, \frac{M_{1} M_{2}}{2}\right)} \\
& =(-1)^{N} \frac{\Gamma\left(M_{1} M_{2}\right) \Gamma\left(-M_{1} M_{2}+1\right)}{\Gamma\left(\frac{M_{1} M_{2}}{2}\right) \Gamma\left(-\frac{M_{1} M_{2}}{2}+1\right)} \simeq \frac{\sin \pi\left(k_{2} \cdot k_{4}\right)}{\sin \pi\left(k_{1} \cdot k_{2}\right)}
\end{aligned}
$$

where, in the nonrelativistic limit, we have

$$
\begin{aligned}
& k_{1} \cdot k_{2} \simeq-M_{1} M_{2}, \\
& k_{2} \cdot k_{4} \simeq \frac{\left(M_{1}+M_{2}\right) M_{2}}{2} .
\end{aligned}
$$

We thus have ended up with a consistent nonrelativistic string BCJ relations. We stress that the above relation is the stringy generalization of the massless field theory BCJ relation [31] to the higher spin stringy particles.

\section{The associate symmetry group of string scattering amplitudes}

In the Lie group approach of special functions, the associate Lie group for the Lauricella function $F_{D}^{(K)}$ in the SSA at each fixed $K$ is the $S L(K+3, \mathbb{C})$ group [30] which contains the $S L(2, \mathbb{C})$ fundamental representation of the $3+1$ dimensional spacetime Lorentz group $\mathrm{SO}(3,1)$. So $\operatorname{sl}(K+3, \mathbb{C})$ contains the $2+1$ dimensional so $(2,1)$ Lorentz spacetime symmetry on the scattering plane in our case as well. In the Regge limit, the Lauricella function in the SSA reduces to the Appell function $F_{1}$ with associate group $S L(5, \mathbb{C})[28]$, which is $K$ independent. In the low energy nonrelativistic limit, the Lauricella function in the $\mathrm{SSA}$ reduces to the Gauss hypergeometric function ${ }_{2} F_{1}$ with associate group $S L(4, \mathbb{C})[30]$, which is also $K$ independent.

In sum, we have identified the associate exact $S L(K+3, \mathbb{C})$ symmetry of string scattering amplitudes with three tachyons and one arbitrary string states of $26 D$ bosonic open string theory. However, since not all Lauricella functions $F_{D}^{(K)}$ with arbitrary independent arguments can be used to represent SSA, it remained to be studied how the basis states of each $\mathrm{SL}(K+3, \mathbb{C})$ group representation for a given $K$ relates to SSA. This important issue is currently under investigation.

Finally, with the $S L(K+3, \mathbb{C})$ group and the recurrence relations of the Lauricella functions $F_{D}^{(K)}$, one can derive infinite number of recurrence relations of SSA of different string states which are valid for all energies, as long as all the Lauricella functions $F_{D}^{(K)}$ in 
the recurrence relation representing the SSA. For a simple example, the following recurrence relation of $F_{D}^{(K)}$ can be verified

$$
c F_{D}^{(K)}\left(b_{j} ; c\right)+c\left(x_{j}-1\right) F_{D}^{(K)}\left(b_{j}+1 ; c\right)+(a-c) x_{j} F_{D}^{(K)}\left(b_{j}+1 ; c+1\right)=0,
$$

where $F_{D}^{(K)}\left(b_{j} ; c\right)$ is the abbreviation for the Lauricella function $F_{D}^{(K)}\left(a ; b_{1}, \ldots, b_{K} ; c ; x_{1}, \ldots, x_{K}\right)$. Only relevant arguments of the recurrence relation are listed for conciseness.

Eq. (6.13) leads to the recurrence relation of SSA

$$
\left(\frac{u}{2}+2-N\right) A_{s t}^{\left(r_{n}^{T}, r_{m}^{P}, r_{l}^{L}\right)}-\left(\frac{s}{2}+1\right) k_{3}^{T} A_{s t}^{\left(r_{n}^{\prime T}, r_{m}^{P}, r_{l}^{L}\right)}=0
$$

where $\left(r_{n}^{\prime T}, r_{m}^{P}, r_{l}^{L}\right)$ means the group $\left(-\left\{r_{1}^{T}-1\right\}^{1},\left\{-r_{2}^{T}\right\}^{2}, \cdots,\left\{-r_{n}^{T}\right\}^{n} ; R_{m}^{P}, R_{l}^{L}\right)$ of polarizations. In eq. (6.13), we have omitted those arguments of $F_{D}^{(K)}$ which remain the same for all three Lauricella functions.

\section{Acknowledgments}

J.C. would like to thank H. Kawai for crucial suggestions of some results of this work. This work is supported in part by the Ministry of Science and Technology and S.T. Yau center of NCTU, Taiwan.

Open Access. This article is distributed under the terms of the Creative Commons Attribution License (CC-BY 4.0), which permits any use, distribution and reproduction in any medium, provided the original author(s) and source are credited.

\section{References}

[1] D.J. Gross and P.F. Mende, The High-Energy Behavior of String Scattering Amplitudes, Phys. Lett. B 197 (1987) 129 [inSPIRE].

[2] D.J. Gross and P.F. Mende, String Theory Beyond the Planck Scale, Nucl. Phys. B 303 (1988) 407 [INSPIRE].

[3] D.J. Gross, High-Energy Symmetries of String Theory, Phys. Rev. Lett. 60 (1988) 1229 [INSPIRE].

[4] D.J. Gross and J.R. Ellis, Strings at superplanckian energies: in search of the string symmetry, Phil. Trans. Roy. Soc. Lond. A 329 (1989) 401.

[5] D.J. Gross and J.L. Manes, The High-energy Behavior of Open String Scattering, Nucl. Phys. B 326 (1989) 73, section 6 [INSPIRE].

[6] C.-T. Chan and J.-C. Lee, Stringy symmetries and their high-energy limits, Phys. Lett. B 611 (2005) 193 [hep-th/0312226] [INSPIRE].

[7] J.-C. Lee, Stringy symmetries and their high-energy limit, hep-th/0303012 [INSPIRE].

[8] C.-T. Chan and J.-C. Lee, Zero norm states and high-energy symmetries of string theory, Nucl. Phys. B 690 (2004) 3 [hep-th/0401133] [InSPIRE]. 
[9] C.-T. Chan, P.-M. Ho and J.-C. Lee, Ward identities and high-energy scattering amplitudes in string theory, Nucl. Phys. B 708 (2005) 99 [hep-th/0410194] [INSPIRE].

[10] C.-T. Chan, P.-M. Ho, J.-C. Lee, S. Teraguchi and Y. Yang, High-energy zero-norm states and symmetries of string theory, Phys. Rev. Lett. 96 (2006) 171601 [hep-th/0505035] [INSPIRE].

[11] C.-T. Chan, P.-M. Ho, J.-C. Lee, S. Teraguchi and Y. Yang, Solving all 4-point correlation functions for bosonic open string theory in the high energy limit, Nucl. Phys. B 725 (2005) 352 [hep-th/0504138] [INSPIRE].

[12] C.-T. Chan, J.-C. Lee and Y. Yang, High energy scattering amplitudes of superstring theory, Nucl. Phys. B 738 (2006) 93 [hep-th/0510247] [INSPIRE].

[13] J.-C. Lee, New Symmetries of Higher Spin States in String Theory, Phys. Lett. B 241 (1990) 336 [INSPIRE].

[14] J.-C. Lee, Decoupling of Degenerate Positive Norm States in String Theory, Phys. Rev. Lett. 64 (1990) 1636 [INSPIRE].

[15] J.-C. Lee and B.A. Ovrut, Zero Norm States and Enlarged Gauge Symmetries of Closed Bosonic String in Background Massive Fields, Nucl. Phys. B 336 (1990) 222 [INSPIRE].

[16] N. Moeller and P.C. West, Arbitrary four string scattering at high energy and fixed angle, Nucl. Phys. B 729 (2005) 1 [hep-th/0507152] [INSPIRE].

[17] P.C. West, A Brief Review of the Group Theoretic Approach to String Theory, in Conformal Field Theories and Related Topics, proceedings of Third Annecy Meeting on Theoretical Physics, LAPP, Annecy le Vieux France, P. Binutruy, P. Sorba and R. Stora eds., North Holland (1988) [Nucl. Phys. B (Proc. Suppl.) 5B (1988) 217].

[18] E. Witten, Perturbative gauge theory as a string theory in twistor space, Commun. Math. Phys. 252 (2004) 189 [hep-th/0312171] [INSPIRE].

[19] R. Radu, M. Spradlin and A. Volovich, Scattering amplitudes in gauge theories: progress and outlook, J. Phys. A 44 (2011) 450301.

[20] G.W. Moore, Finite in all directions, hep-th/9305139 [INSPIRE].

[21] G.W. Moore, Symmetries of the bosonic string S matrix, hep-th/9404025 [INSPIRE].

[22] C.-T. Chan, S. Kawamoto and D. Tomino, To see Symmetry in a Forest of Trees, Nucl. Phys. B 885 (2014) 225 [arXiv: 1309.3443] [InSPIRE].

[23] J.-C. Lee and Y. Yang, Review on High energy String Scattering Amplitudes and Symmetries of String Theory, arXiv:1510.03297 [INSPIRE].

[24] S.L. Ko, J.-C. Lee and Y. Yang, Patterns of high energy massive string scatterings in the Regge regime, JHEP 06 (2009) 028 [arXiv:0812.4190] [INSPIRE].

[25] J.-C. Lee and Y. Mitsuka, Recurrence relations of Kummer functions and Regge string scattering amplitudes, JHEP 04 (2013) 082 [arXiv:1212.6915] [INSPIRE].

[26] J.-C. Lee and Y. Yang, The Appell function $F_{1}$ and Regge string scattering amplitudes, Phys. Lett. B 739 (2014) 370 [arXiv:1406.1285] [InSPIRE].

[27] J.-C. Lee, C.H. Yan and Y. Yang, High energy string scattering amplitudes and signless Stirling number identity, SIGMA 8 (2012) 045 [arXiv: 1012.5225] [INSPIRE].

[28] W. Miller Jr., Lie theory and the Appell functions $F_{1}$, SIAM J. Math. Anal. 4 (1973) 638. 
[29] S.H. Lai, J.-C. Lee and Y. Yang, The string BCJ relations revisited and extended recurrence relations of nonrelativistic string scattering amplitudes, JHEP 05 (2016) 186 [arXiv: 1601.0381] [INSPIRE].

[30] W. Miller Jr., Lie theory and generalizations of the hypergeometric functions, SIAM J. Appl. Math. 25 (1973) 226.

[31] Z. Bern, J.J.M. Carrasco and H. Johansson, New Relations for Gauge-Theory Amplitudes, Phys. Rev. D 78 (2008) 085011 [arXiv: 0805.3993] [InSPIRE].

[32] D. Oprisa and S. Stieberger, Six gluon open superstring disk amplitude, multiple hypergeometric series and Euler-Zagier sums, hep-th/0509042 [INSPIRE].

[33] H. Kawai, private conversations.

[34] N.E.J. Bjerrum-Bohr, P.H. Damgaard and P. Vanhove, Minimal Basis for Gauge Theory Amplitudes, Phys. Rev. Lett. 103 (2009) 161602 [arXiv:0907.1425] [InSPIRE].

[35] S. Stieberger, Open Éamp; Closed vs. Pure Open String Disk Amplitudes, arXiv:0907.2211 [INSPIRE].

[36] J. Kampe de Feriet and P. Appell, Fonctions hypergeometriques et hyperspheriques, Gauthier-Villars, Paris (1926).

[37] C.-T. Chan, J.-C. Lee and Y. Yang, Notes on high energy bosonic closed string scattering amplitudes, Nucl. Phys. B $\mathbf{7 4 9}$ (2006) 280 [hep-th/0604122] [INSPIRE].

[38] H. Kawai, D.C. Lewellen and S.H.H. Tye, A Relation Between Tree Amplitudes of Closed and Open Strings, Nucl. Phys. B 269 (1986) 1 [inSPIRE]. 